\title{
Development and Evaluation of Biodegradable Chitosan Films of Metronidazole and Levofloxacin for the Management of Periodontitis
}

\author{
Gayasuddin Khan, ${ }^{1}$ Sarita K. Yadav, ${ }^{1}$ Ravi R. Patel, ${ }^{1}$ Gopal Nath, ${ }^{2}$ Monika Bansal, ${ }^{3}$ and Brahmeshwar Mishra ${ }^{1,4}$
}

Received 11 August 2015; accepted 3 December 2015; published online 21 December 2015

\begin{abstract}
Metronidazole (MZ) and levofloxacin (LF) are widely employed for treatment of periodontitis, but high oral dose and resistance development after long-term oral administration limit their use. The aim of this study was to alleviate shortcomings in the treatment of periodontitis by fabrication of intrapocket, biodegradable films of chitosan (CS) loaded with MZ and LF meant for inserting into periodontal pockets to treat infections. The films were developed by solvent casting technique using propylene glycol as plasticizer and glutaraldehyde as crosslinking agent. Their physical characteristics, such as drug content, surface $\mathrm{pH}$, swelling index, and folding endurance, exhibited results within limit. Further, FTIR and DSC studies revealed stability of films and compatibility between drugs and excipients. SEM images of films showed the presence of free drug particles on the surface causing burst effect. In vitro release in McIlvaine buffer $\mathrm{pH} 6.6$ was of sustained nature assisted by the burst effect. CS and crosslinking agent concentrations negatively affected drug release and positively affected $\mathrm{T}_{90}$ (time for releasing $90 \%$ of the drug) due to altered matrix density. In contrast, the plasticizer concentration increases membrane permeability and hence increased drug release, lowering $\mathrm{T}_{90}$. Crosslinked films demonstrated sustained release up to 7 days. The antibacterial efficacy of films was tested on Staphylococcus aureus and Escherichia coli, indicating good antibacterial activity. Clinical trials on patients proved the therapeutic efficacy of the films by a significant $(p<0.05)$ decrease in the clinical markers of periodontitis, i.e. gingival index, plaque index and pocket depth. Conclusively, the films of MZ and LF were successful tools for the management of periodontitis.
\end{abstract}

KEY WORDS: chitosan; clinical assessment; film; levofloxacin; metronidazole.

\section{INTRODUCTION}

The most serious consequences of bad oral hygiene are the development of chronic oral inflammatory diseases like periodontitis that may extend systemically and affect diabetes mellitus, cardiovascular disease and infective endocarditis, etc. $(1,2)$. Periodontal disease is a pathological condition characterized by inflammation and degeneration of the gums (gingival), supporting bone (alveolar bone), periodontal ligament and cementum initiated by the anaerobic and microaerophilic microorganisms that colonize the tooth surface and infect the surroundings (3).

Current periodontal therapy is practiced with an aim to cure the inflamed tissue, to eliminate the depth of the diseased pockets, to reduce the number of pathogenic bacteria and to stop bone resorption with mechanical cleaning such as scaling and root planing (SRP) as well as systemic or local

\footnotetext{
${ }^{1}$ Department of Pharmaceutics, Indian Institute of Technology, Banaras Hindu University, Varanasi, 221005, Uttar Pradesh, India.

${ }^{2}$ Department of Microbiology, Institute of Medical Sciences, Banaras Hindu University, Varanasi, 221005, India.

${ }^{3}$ Faculty of Dental Sciences, Institute of Medical Sciences, Banaras Hindu University, Varanasi, 221005, India.

${ }^{4}$ To whom correspondence should be addressed. (e-mail: bmishrabhu@rediffmail.com)
}

administration of antimicrobial agents. Although systemic administration of antibiotics is beneficial, it requires high oral doses to achieve effective concentrations in the gingival crevicular fluid (GCF), and long-term administration may lead to the development of resistance. Whereas local application of mouth rinses, gels, and toothpastes require less dose but controls only supragingival plaques and mucosal infections. Moreover, such local delivery systems also require high initial concentration and multiple applications in order to provide sustained effectiveness (4).

To circumvent the drawbacks associated with systemic as well as localized drug delivery, controlled release devices meant for intrapocket administration have been proposed which can directly target the microbes proliferating in the pockets $(5,6)$. The desirable features for intrapocketlocalized drug delivery are mucoadhesivity, biodegradability, biocompatibility, non-toxicity and providing controlled drug delivery for prolonged time. All these features can be met by films based on chitosan (CS) loaded with selected antimicrobials as proposed in the present study.

CS, a natural polymer obtained by alkaline deacetylation of chitin, is nontoxic, biocompatible, biodegradable and moreover metabolized by certain human enzymes, especially lysozyme. These properties make chitosan a good candidate for conventional as well as novel drug delivery systems $(7,8)$. 
Further, it can be easily casted into film by using solvent casting method (9). It acts as a promising matrix for controlled and sustained drug release and possesses excellent filmforming properties (10). In addition to cost effectiveness, its biodegradable nature has caught more attraction and made it a high patient-compliant drug delivery device for periodontitis. The device dissolves within the pocket, and there is no need of the patient to revisit clinic for removal of the device. Further, local intrapocket administration of such devices requires less dose, little or no systemic uptake, and therefore minimized side effects of loaded antibiotics (3).

In combination therapy, selection of drugs should be made such that it targets a wide range of proliferating bacteria. Metronidazole (MZ) is considered as a front-line chemotherapeutic agent and is selectively active against obligate anaerobic microbes residing into inflamed periodontal pockets. Also, therapeutic concentration is attained early as it has lower minimum inhibitory concentration (MIC) for the treatment and management of periodontitis $(11,12)$. In addition to MZ, levofloxacin (LF) has been used due to its activity against facultative anaerobic periodontopathic bacteria. LF is an active isomer of ofloxacin which is twice active in comparison to ofloxacin and widely used for the treatment of periodontal diseases (13). The combination of MZ and LF was selected to target wide range of periodontal pathogens and effective management of disease. Furthermore, the rationale of the combination of LF and MZ is based on the fact that quinolones and imidazole derivatives act synergistically. Credito et al. and Bharadwaj et al. reported that the combination of LF with MZ showed synergistic action against periodontopathic pathogens $(14,15)$.

In order to achieve high local bioactivity and low systemic side effects of antibiotics in the treatment of periodontal infections, a localized controlled delivery system is desirable. Therefore, the present study was performed to fabricate CS films containing MZ and LF to achieve controlled and sustained drug concentration above MIC for a prolonged period. The localized administration of MZ and LF could be advantageous for the improvement of periodontal condition in patients in terms of targeting the pathogens in the pockets and decreasing systemic side effects and healing time simultaneously leading to quick regeneration of the destructed tissues. The films were prepared by simple solvent casting technique without use of any harmful organic solvents. The prepared films were characterized for physical parameters and stability. The films with desired quality traits can be obtained by considering the effect of various formulation variables. The antibacterial efficacy of films was evaluated on selected strains of Staphylococcus aureus (ATCC25323) and Escherichia coli (ATCC25922). Subsequently, preliminary clinical study on the optimized formulations was performed in an attempt to prove their efficacy. All the treatments for periodontitis were done as an adjunct to SRP.

\section{MATERIALS AND METHODS}

\section{Materials}

MZ and LF were generous gifts by Nicholas Piramal (Mumbai, India) and Glenmark Pharmaceuticals (Baddi, India), respectively. Chitosan (medium molecular weight,
90\% deacetylation), propylene glycol, glutaraldehyde, acetic acid, citric acid monohydrate and disodium monohydrate orthophosphate were purchased from Sigma Aldrich. Millipore Direct $Q \circledR 3 U V$ water was used in all the studies.

\section{Methods}

\section{Fabrication of Films}

Periodontal films were prepared by solvent casting method (11). The films were prepared as per formula given in Table I. Accurately weighed quantity of CS was dissolved in $40 \mathrm{ml}$ of $0.5 \%$ acetic acid solution and stirred for $24 \mathrm{~h}$ for complete solubilization. The weighed amount of LF and MZ was incorporated in the polymer solution and stirred for $6 \mathrm{~h}$. A measured quantity of propylene glycol (as a plasticizer) was added. After deaerating under vacuum for $6 \mathrm{~h}$, the solution was poured on levelled glass mould having size of $5 \times 4 \times 1.2 \mathrm{~cm}$ and placed in an oven maintained at $50^{\circ} \mathrm{C}$ (Tempo Industrial Corporation, Mumbai). The system was left undisturbed for $24 \mathrm{~h}$ to allow complete evaporation. The formed films were completely removed from the glass mould and punched out in desired size, wrapped in aluminium foil and stored in desiccators until further use. For crosslinking, the films were dipped into the solution of crosslinking agent glutaraldehyde for $24 \mathrm{~h}$ and then removed and dried. Placebo films without drug containing only CS and plasticizer were prepared as control.

\section{Fourier Transform Infrared Spectroscopic Studies}

Fourier transform infrared (FTIR) spectra of samples were taken on Shimadzu (model 8400S, Tokyo, Japan) instrument to investigate the possible interaction between the drug and excipients. The samples were crushed with $\mathrm{KBr}$ to get the pellets by applying a pressure of $300 \mathrm{~kg} \mathrm{~cm}^{-2}$. FTIR spectra of pure drug and polymer and the physical mixture of drug and polymer in ratio of $1: 1$ and films were scanned in the range between 4000 and $400 \mathrm{~cm}^{-1}$.

\section{Differential Scanning Calorimetric Studies}

The compatibility and physical state of drugs inside the optimized formulation (F-11) were determined by differential scanning calorimetric (DSC). Thermograms were used with a differential scanning calorimeter (Mettler, Toledo, 822e). The instrument was calibrated with $5 \mathrm{mg}$ of indium at a heating rate of $10^{\circ} \mathrm{C} \mathrm{min}^{-1}$ (peak maximum $175.6^{\circ} \mathrm{C}$ for $\mathrm{LF}$ and $190^{\circ} \mathrm{C}$ for $\mathrm{MZ}$ ). The thermal behaviour was studied by heating 2$10 \mathrm{mg}$ of samples heating at a rate of $10^{\circ} \mathrm{C} \mathrm{min}^{-1}$ from $25^{\circ} \mathrm{C}$ to $300^{\circ} \mathrm{C}$ in a hermetically sealed pan with a pinhole in the lid under a nitrogen purge of $20 \mathrm{ml} \mathrm{min}^{-1}$.

\section{Physical Characterization of Periodontal Films}

Thickness and Weight Variation. The thickness of the films was measured by using a screw gauge. Three randomly selected films of each formulation having surface area $1 \mathrm{~cm}^{2}$ were used. For determination of weight variation, 10 patches of each formulation are weighed separately on electronic balance (Shimadzu, Japan) and the average weight of the 
Table I. Composition of Different Batches of Film

\begin{tabular}{|c|c|c|c|c|c|}
\hline \multirow[t]{2}{*}{ Batch no. } & \multirow[t]{2}{*}{ Chitosan (g) } & \multirow[t]{2}{*}{ Propylene glycol (g) } & \multirow[t]{2}{*}{ Glutaraldehyde (g) } & \multicolumn{2}{|c|}{ Drugs (g) } \\
\hline & & & & $\mathrm{LF}$ & $\mathrm{MZ}$ \\
\hline F-1 & 0.6 & 0.12 & - & 0.1 & 0.1 \\
\hline $\mathrm{F}-2$ & 0.6 & 0.18 & - & 0.1 & 0.1 \\
\hline F-3 & 0.6 & 0.24 & - & 0.1 & 0.1 \\
\hline F-4 & 0.7 & 0.28 & - & 0.1 & 0.1 \\
\hline F-5 & 0.7 & 0.21 & - & 0.1 & 0.1 \\
\hline F-6 & 0.7 & 0.14 & - & 0.1 & 0.1 \\
\hline F-7 & 0.8 & 0.16 & - & 0.1 & 0.1 \\
\hline F-8 & 0.8 & 0.24 & - & 0.1 & 0.1 \\
\hline F-9 & 0.8 & 0.32 & - & 0.1 & 0.1 \\
\hline F-10 & 0.8 & 0.16 & 0.02 & 0.1 & 0.1 \\
\hline F-11 & 0.8 & 0.16 & 0.04 & 0.1 & 0.1 \\
\hline
\end{tabular}

patch was calculated. The individual values can be varied within $\pm 5 \%$ of the mean (16).

Uniformity of Drug Content. The uniformity of the drug content, between batches and within batches, was determined by dissolving individually weighed films $(n=3)$ in a volumetric flask containing $5 \mathrm{ml}$ of glacial acetic acid. After gentle stirring for an hour, the volume was made up to $100 \mathrm{ml}$ with McIlvaine buffer $\mathrm{pH}$ 6.6. The resultant solution was filtered through a G-2 glass filter $(40 \mu \mathrm{m})(17)$. Following adequate dilutions, the filtrate was analyzed for MZ and LF contents using simultaneous equation method in following equations:

$\mathrm{CMZ}=\frac{A 1 a y 2-A 2 a y 1}{\operatorname{ax} 1 a y 2-a x 2 a y 1}$

$\mathrm{CLF}=\frac{A 1 a \times 2-A 2 a x 1}{\operatorname{ax1ax2-a\times 2ax1}}$

The $\lambda_{\max }$ for MZ and LF was obtained at 319 and $287 \mathrm{~nm}$ using UV spectrophotometer (Shimadzu-1700, Japan). In the above equations, A1 and A2 are the absorbance of samples at 319 and $287 \mathrm{~nm}$ respectively, ax1 and $a \times 2$ are absorptivities of $\mathrm{MZ}$ at $\lambda 1$ and $\lambda 2$, respectively, and $a y 1$ and $a y 2$ are absorptivities of LF at $\lambda 1$ and $\lambda 2$, respectively. CMZ and CLF are the concentrations of MZ and LF respectively.

Surface $p H$. For determination of surface $\mathrm{pH}$, periodontal films were allowed to swell for $2 \mathrm{~h}$ on the surface of agar plates prepared in McIlvaine buffer $\mathrm{pH}$ 6.6. The surface $\mathrm{pH}$ was determined using $\mathrm{pH}$ paper (Qauligens Fine Chemicals, Galaxo India Limited, India) placed on the surface of the wetted patch. A mean of the three readings was recorded (18).

Folding Endurance. The folding endurance or flexibility of the film was determined by repeatedly folding the film at $180^{\circ}$ angle of the plane at the same place until it breaks or folded to 300 times without breaking. The number of times the films folded without breaking as considered as folding endurance (16).

Swelling Index. The swelling index was calculated to study the hydration characteristics of the films. After drying in an oven, the $1 \times 1 \mathrm{~cm}$ films were weighed $(W 1)$ and allowed to swell in petri dish $(3.5 \mathrm{~cm}$ diameter containing $5 \mathrm{ml}$ McIlvaine buffer ( $\mathrm{pH} \mathrm{6.6)}$. At predefined intervals up to $24 \mathrm{~h}$, the swollen films were reweighed (W2) after drying the excess of water from the surface of films using filter paper (19). The swelling index of each system was calculated using the following equation:

$\%$ Swelling index $=\frac{W 2-W 1}{W 1} \times 100$

\section{Scanning Electron Microscopic Studies}

The surface morphology of the drug-loaded film was examined by scanning electron microscopy (SEM) (FEI, Quanta 200F, Japan). The film sample was mounted on metal stubs with double-sided adhesive band then gold was sputtered on the specimen to ensure sufficient electrical conductivity. The images were taken using environmental mode and ET detector with $10 \mathrm{kV}$ excitation energy.

\section{In Vitro Drug Release Studies}

The medicated films were cut into circular shape with diameter of $5 \mathrm{~mm}$ and placed in $5 \mathrm{ml}$ vial containing $2 \mathrm{ml}$ McIlvaine buffer $\mathrm{pH} 6.6$, previously warmed at $37^{\circ} \mathrm{C}$, without shaking, to simulate the stagnant intrapocket condition (20). At predetermined time intervals 1, 2, 4, 6, 8, 12, 24, 48, 72, 96, 120,144 , and $168 \mathrm{~h}$, the whole release medium was withdrawn and replaced with an equal volume of previously warmed fresh buffer solution. The withdrawn sample was analyzed for LF and MZ contents by simultaneous equation method as described earlier. Release media of placebo film was used as blank to circumvent any interference from polymer used. The results were reported as an average of three determinations (21). To understand the mechanism of drug release, correlation coefficients $\left(r^{2}\right)$ and release rate constants $(K)$ for various models (zero order, first order, Higuchi model and Korsemeyer-Peppas model) were determined for all batches by Microsoft Excel 2007. The release model having $r^{2}$ value close to 1 was considered as the best fit model. 


\section{Stability Studies}

The films were wrapped individually in butter paper followed by aluminium foil and stored at $30^{\circ} \mathrm{C} \pm 2{ }^{\circ} \mathrm{C} / 65 \% \pm$ $5 \% \mathrm{RH}$ for 90 days. The samples were analyzed for physical changes such as colour, texture and other physical parameters. The FTIR spectra of stored films were compared with the freshly prepared films.

\section{In Vitro Antibacterial Activity of Periodontal Films}

The fabricated placebo films, LF films and optimized LF + MZ films were tested for their antibacterial activity against $S$. aureus (ATCC25323) and E. coli (ATCC25922) using disc diffusion method (22). Briefly, Muller Hinton agar (MHA) plates were used for screening, prepared by pouring $15 \mathrm{ml}$ of molten media into sterile petri plates. Then $1.0 \mathrm{ml}$ of 18 -h cultured bacteria adjusted to $0.5 \mathrm{McF}$ arland standards in sterile saline to achieve concentration of $10^{7} \mathrm{CFU} \mathrm{ml}^{-1}$ was spread on the surface of MHA agar plates with the help of sterile swab sticks. The discshaped polymer film of 5-mm diameter was then placed on the surface of the medium and incubated at $37^{\circ} \mathrm{C}$ for $24 \mathrm{~h}$. At the end of incubation, the inhibition zones were examined around the polymer disc film which, if present, were measured with transparent ruler in millimetres. This film was then transferred after every $24 \mathrm{~h}$ into another swabbed sterile MHA plates, and the zone of inhibition was measured. The study was performed in triplicate.

\section{Clinical Study}

The single-blind clinical study was conducted in the Faculty of Dental Sciences, Institute of Medical Sciences, Banaras Hindu University, India, after obtaining approval from the ethical committee of the Institute [Dean/2012-13/ 133/5-10 July]. Ten patients (20-50 years) of either sex were recruited from the outpatient department at the Department of Dentistry, Sir Sunder Lal Hospital, IMS (BHU). The patients were first screened for a sign of chronic periodontitis based on following inclusion and exclusion criteria:

\section{Inclusion Criteria.}

1) Signed informed consent form

2) Patients free from systemic diseases

3) No pregnant or lactating women

4) Age between 20 and 50 years

5) Demonstrate bleeding on probing from the base of the pocket

6) Patients suffering from chronic periodontitis, with pocket depth $\geq 5 \mathrm{~mm}$ in at least 4 sites in one patient

7) No periodontal therapy in the last 6 months

8) No antimicrobials for 1 month prior to the study

\section{Exclusion Criteria.}

1) Poor oral hygiene maintenance during the treatment

2) Allergic history to any drug

3) Habits like smoking and pan chewing

Study Design. In the present study, the four sites in each patient were taken in the study so as a total of 40 periodontal sites from 10 patients were included for evaluation. These four periodontal sites in each patient were randomly and blindly divided into the following four groups: i) group 1-SRP; ii) group 2-SRP + placebo film; iii) group 3-SRP with film containing LF; iv) group 4-SRP with film containing LF and MZ.

Periodontal disease is a multifactorial disease in nature, and the progression of periodontal disease and outcome of the treatment depend upon the interaction between the microorganisms and host-related factors. Therefore, all treatments were given in one patient at four sites to avoid the influence of many risk factors including genetic, socioeconomic status, age, gender, oral hygiene maintenance, host immune defence mechanism and stress related to periodontal disease on the outcomes of the treatment provided.

Clinical Parameters. Clinical parameters such as gingival index (GI), bleeding on probing (BOP), probing pocket depth (PPD) and clinical attachment level (CAL) were determined in the study. GI and BOP indices were recorded at the baseline before the treatment and at first week, second week, fourth week and eighth week after treatment. PPD and CAL were recorded at baseline before treatment and fourth week and eighth week after treatment using the UNC-15 (Hu-Friedy, USA) periodontal probe.

Gingival Index

Score- Criteria

0 Normal gingiva

1. Mild inflammation, slight change in colour, slight edema, no bleeding on probing

2. Moderate inflammation, redness, edema and glazing, bleeding on probing

3. Severe inflammation, marked redness and edema, ulcerations, tendency to spontaneous bleeding

Bleeding on Probing To test for BOP, the probe was carefully introduced parallel to the long axis of the tooth to the bottom of the pocket and gently moved laterally along the pocket wall. BOP was recorded after 30 to $60 \mathrm{~s}$ of insertion of the periodontal probe.

Score- Criteria

0 . No bleeding

1. Bleeding on probing

Probing Pocket Depth PPD was measured from the crest of the gingival margin to the base of the pocket.

Clinical Attachment Level CAL was measured from the cementoenamel junction (CEJ) to the base of the pocket.

Periodontal Therapy. Complete gingival and periodontal examination was carried out before starting the treatment. Then, all patients underwent for full mouth supra- and subgingival scaling and root planning. After scaling and root planning, the selected periodontal sites of groups 2, 3 and 4 received the respective treatment. The periodontal site of group 1 was used as control and did not receive any film. Before insertion of the film into the periodontal pocket, it was sterilized by autoclaving (23). Periodontal dressing (Coe-pack) was used to secure the film in place. After 1 week, 
periodontal dressing was removed. All recruited patients were recalled at the scheduled follow up i.e. first week, second week, fourth week and eighth week for recording the clinical parameters and evaluating the oral hygiene status. Each patient was asked for discomfort or allergy occurring after placement of the film and instructed to avoid the hard or sticky food to keep away from gingival injury (24).

Statistical Analysis. Mean \pm standard deviation (SD) was calculated for the observed values in each experimental group from clinical study. Statistical analysis was performed by twoway analysis of variance (ANOVA) followed by Bonferroni post tests. GraphPad Prizm 5.03 (Graph Pad Software Inc., USA) software was used for statistical analysis and graph preparation.

\section{RESULTS AND DISCUSSION}

\section{Fourier Transform Infrared Spectroscopic Studies}

The samples were scanned in the region of $4000-400 \mathrm{~cm}^{-1}$ for FTIR studies (Figs. 1 and 2). Pure MZ showed characteristic peaks at $1265.28 \mathrm{~cm}^{-1}$ (C-O stretching), $1369.22 \mathrm{~cm}^{-1}$ (N-O stretching), $1536.23 \mathrm{~cm}^{-1}$ ( $\mathrm{C}=\mathrm{N}$ stretching) and $3223.2 \mathrm{~cm}^{-1}$ (OH) (Fig. 1a). Characteristic peaks at $1723 \mathrm{~cm}^{-1}$ ( $>\mathrm{C}=\mathrm{O}$ of lactum ring), $1884 \mathrm{~cm}^{-1}$ (>C=O of quinolone moiety), $2935 \mathrm{~cm}^{-1}$ (aromatic C-H stretching) and $3275.5 \mathrm{~cm}^{-1}$ (O-H group of carboxyl moiety) were observed for pure LF (Fig. 1b). FTIR spectra of chitosan showed characteristic band at $3450 \mathrm{~cm}^{-1}$ which is attributed to $-\mathrm{NH}_{2}$ and $-\mathrm{OH}$ groups' stretching vibration, and the peak at $1652 \mathrm{~cm}^{-1}$ indicates the presence of amide group (Fig. 1c). Further, carbonyl stretching of chitosan can be seen at $1733 \mathrm{~cm}^{-1}$. Infrared spectra of physical mixture of CS, MZ and
LF showed characteristic peaks of all the excipients and no new bands or shifts in characteristic peaks appeared in any sample, indicating the absence of any possible interaction between the drug and polymer (Fig. 1d). Therefore, it can be speculated that the drugs and polymers are compatible and can be formulated into films.

The spectrum of glutaraldehyde crosslinked films showed absorption bands at $1400 \mathrm{~cm}^{-1}$ (C-H bending) and $1350 \mathrm{~cm}^{-1}$ (O-H bending) (Fig. 2). The bands were rather intense as a consequence of aliphatic C-H absorption (25). Further, the peak at about $1658 \mathrm{~cm}^{-1}$ is reflective of imine bond $(\mathrm{C}=\mathrm{N})$ and the peak at $1595 \mathrm{~cm}^{-1}$ is associated with the $\mathrm{C}=\mathrm{C}$ bond. The imine bond formed between the amine groups of CS and aldehyde groups of the condensation product of glutaraldehyde, while unsaturated $\alpha-\beta$ double bond $(-\mathrm{C}=\mathrm{C}-$ ) was formed due to dehydration and condensation of glutaraldehyde (26). Thus, infrared studies conclude towards compatibility of ingredients employed in fabrication of films with successful crosslinking of films.

\section{Differential Scanning Calorimetric Studies}

DSC studies were performed to characterize the solid state of drugs and polymers. Further, compatibility between drug and excipients can be evaluated by observing the thermal behaviour of compounds such as elimination or appearance of an endothermic or exothermic peak. If all thermal features more or less remain the same, compatibility can be expected. MZ showed sharp endothermic peak at $162.5^{\circ} \mathrm{C}$ attributed to its melting point. The sharp melting peaks exhibited by drugs confirm their existence as crystallite (Fig. 3a). Two endothermic peaks $86.2^{\circ} \mathrm{C}$ (broad) and $223.8^{\circ} \mathrm{C}$ (sharp) were observed in thermogram of $\mathrm{LF}$,

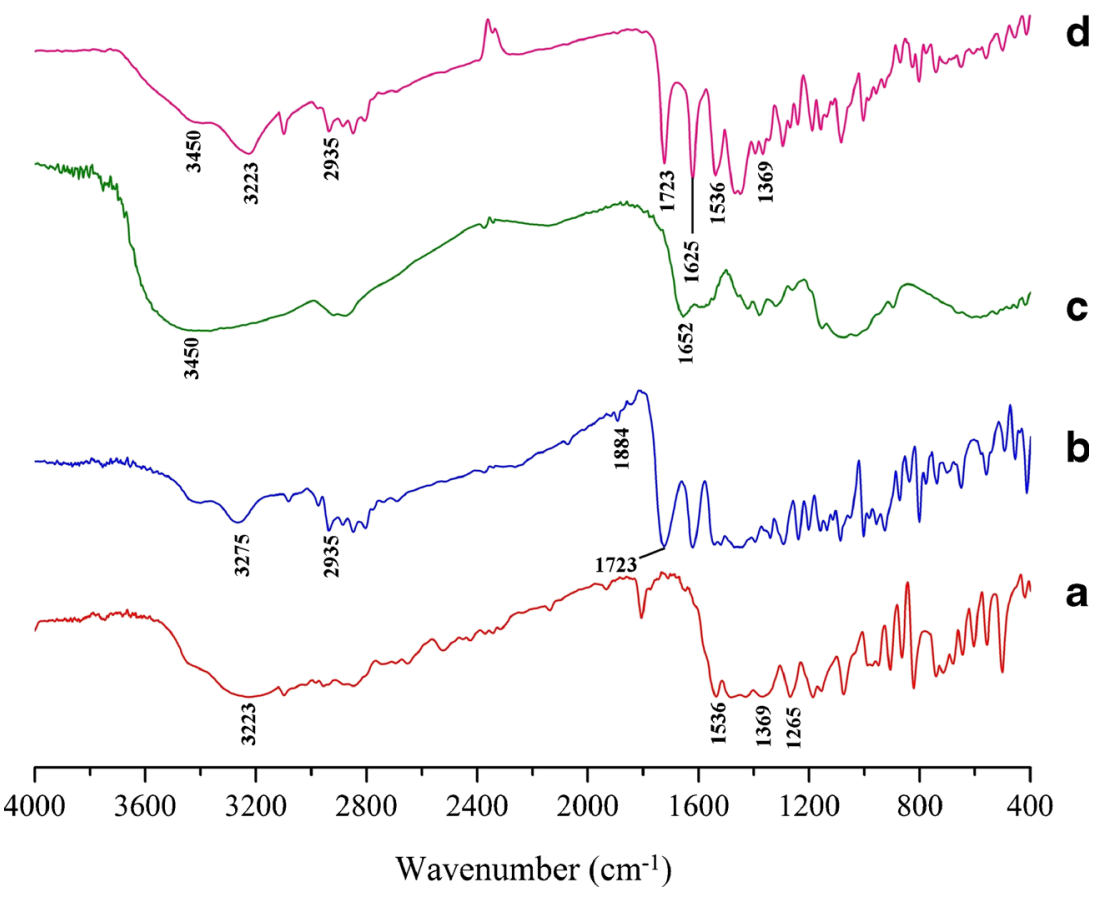

Fig. 1. Infrared spectra of a metronidazole, $\mathbf{b}$ levofloxacin, $\mathbf{c}$ chitosan, $\mathbf{d}$ physical mixture of drugs and polymer 


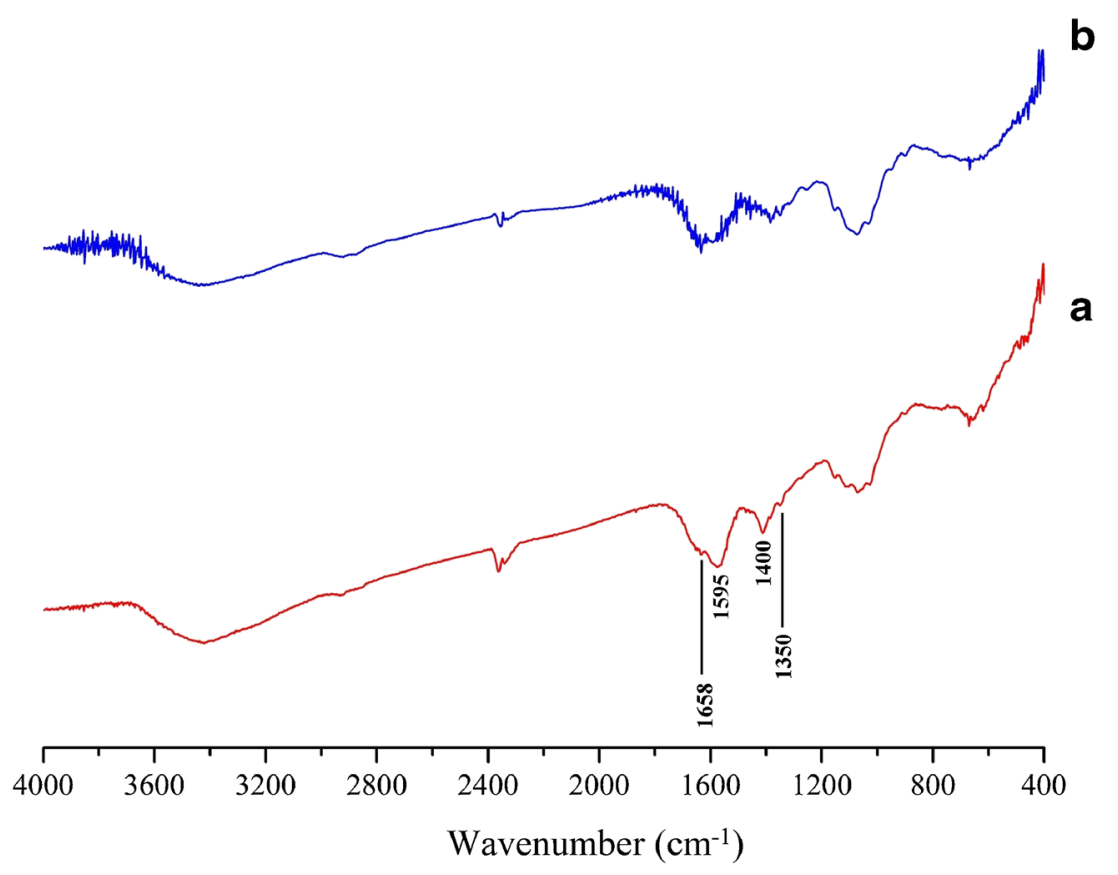

Fig. 2. Infrared spectra of a film (F-11) before storage and $\mathbf{b}$ film after storage

corresponding to dehydration and melting of drug, respectively (Fig. 3b). Apart, CS showed a flat curve due to its amorphous nature (Fig. 3c). Physical mixture of MZ, LF and CS showed the presence of characteristic peaks of drugs, indicating physical compatibility between excipients (Fig. 3d). Instead, formulated periodontal films showed flat curve with no endothermic or exothermic peaks (Fig. 3e). The absence of drugs endothermic peak in the films was observed which may be due to lower concentration of drug relative to polymer that is amorphous in nature or it may be due to reduced crystallinity of drugs during stress conditions of preparation process.

\section{Thickness, Weight and Content Uniformity}

The thickness and weight variation of films are directly associated with the uniformity and accuracy of dosing. The average thickness of all prepared periodontal films F-1 to F-11 ranged from $0.29 \pm 0.05 \mathrm{~mm}$ to $0.35 \pm 0.06 \mathrm{~mm}$ (Table II). Weight variation values varied between $5.96 \pm 0.07 \mathrm{mg}$ and $6.31 \pm 0.08 \mathrm{mg}$ $(n=3)$. Content uniformity studies of the films showed that both the drug were uniformly distributed, and the combined recovery was found to be in the range of $93.01 \%$ to $97.09 \%$ for formulations F-1 to F-11. The obtained values within limit depicted that films of uniform weight, thickness and content could be obtained

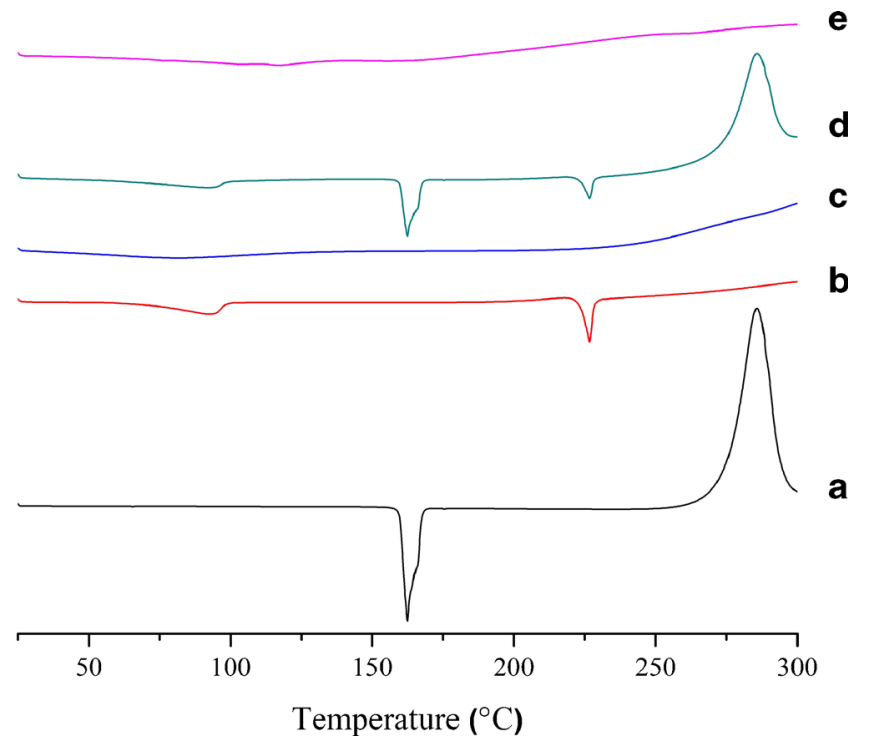

Fig. 3. DSC thermograms of a metronidazole, b levofloxacin, $\mathbf{c}$ chitosan, d physical mixture of drugs and polymer, e film (F-11) 
Table II. Physicochemical Characteristics of Different Batches

\begin{tabular}{lccccc}
\hline Formulation code & Weight $^{\mathrm{a}}(\mathrm{mg} \pm \mathrm{SD})$ & Thickness $^{\mathrm{a}}(\mathrm{mm} \pm \mathrm{SD})$ & Total drug content $^{\mathrm{a}}(\%)$ & Surface pH & Folding endurance \\
\hline F-1 & $6.26 \pm .05$ & $0.29 \pm 0.05$ & $97.40 \pm 2.46$ & 6.0 & 287 \\
F-2 & $6.31 \pm .08$ & $0.32 \pm 0.08$ & $96.50 \pm 2.49$ & 6.0 & 292 \\
F-3 & $6.14 \pm .06$ & $0.35 \pm 0.06$ & $95.20 \pm 3.07$ & 6.0 & 298 \\
F-4 & $6.20 \pm .06$ & $0.32 \pm 0.06$ & $95.70 \pm 2.67$ & 6.5 & 289 \\
F-5 & $5.96 \pm .07$ & $0.30 \pm 0.07$ & $96.24 \pm 2.27$ & 6.5 & 299 \\
F-6 & $6.40 \pm .08$ & $0.32 \pm 0.08$ & $94.19 \pm 2.96$ & 6.5 & $>300$ \\
F-7 & $6.28 \pm .02$ & $0.32 \pm 0.05$ & $95.16 \pm 2.43$ & 7 & $>300$ \\
F-8 & $6.26 \pm .04$ & $0.31 \pm 0.05$ & $94.50 \pm 2.12$ & 7 & $>300$ \\
F-9 & $6.20 \pm .01$ & $0.32 \pm 0.03$ & $93.70 \pm 2.32$ & 7 & $>300$ \\
F-10 & $6.27 \pm .02$ & $0.33 \pm 0.04$ & $94.00 \pm 2.14$ & 7 & 295 \\
F-11 & $6.13 \pm .04$ & $0.34 \pm 0.02$ & $93.80 \pm 2.27$ & 7 & 288
\end{tabular}

$S D$ (standard deviation)

${ }^{a}$ Result is presented as mean $\pm \mathrm{SD}, n=3$

by solvent casting method. The method is reproducible and produces minimum batch variability.

\section{Surface pH}

Surface $\mathrm{pH}$ of films was taken into consideration because too much acidic or basic $\mathrm{pH}$ affects the area of application and causes irritation to oral mucosal membrane leading to patient discomfort (27). In addition, acidic $\mathrm{pH}$ leads to dissolution of enamel and demineralisation of teeth. To overcome these problems, the $\mathrm{pH}$ of the films must be closer to $\mathrm{pH}$ of the GCF ( $\mathrm{pH}$ 6.6). The surface $\mathrm{pH}$ for films F-1 to F-11 ranged from 6 to 7, indicating acceptability of the formulation for pocket insertion. In fact, formulations with $\mathrm{pH} 6.5$ to 7 are more preferred due to closeness of $\mathrm{pH}$ values to neutrality.

\section{Folding Endurance}

The folding endurance was found within acceptable range for all the films. The folding endurance of the films was found to increase with increase in plasticizer concentration. Plasticizer has property to add flexibility to films which will thereby aid easy insertion of the films into pockets. However, incorporation of crosslinking agent (glutaraldehyde) in batches F-10 and F-11 has reduced folding endurance; this observed decrease in flexibility with an increase in the concentration of crosslinking agent may be due to the increase in the extent of crosslinking of the polymeric chains which hinders the free movement of molecules. The concentration of plasticizer was selected at higher concentrations of 20 to $40 \%$ on the basis of earlier reports which demonstrate good plasticization effect of propylene glycol. However, at lower concentrations below $20 \%$, it does not act as plasticizer. This phenomenon is reported as "antiplasticization effect" by some researchers $(28,29)$. The effect has been explained as at lower concentrations propylene glycol forms strong interaction with the polymer due to its crosslinking nature, which decreases the molecular mobility of the polymer resulting into decreased folding endurance.

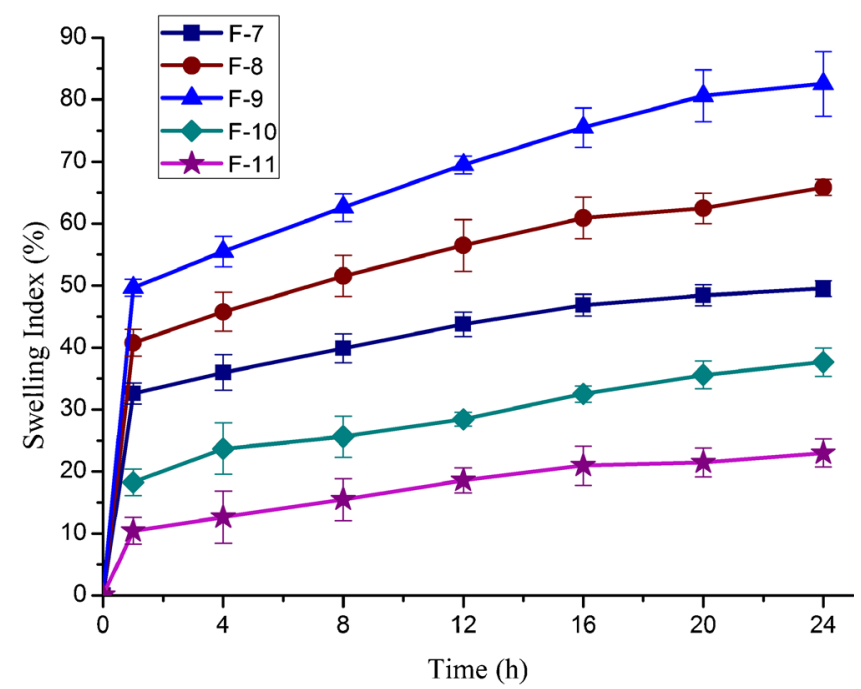

Fig. 4. Histograms representing percent swelling index of selected batches (vertical bars represent mean $\pm \mathrm{SD}, n=3$ ) 

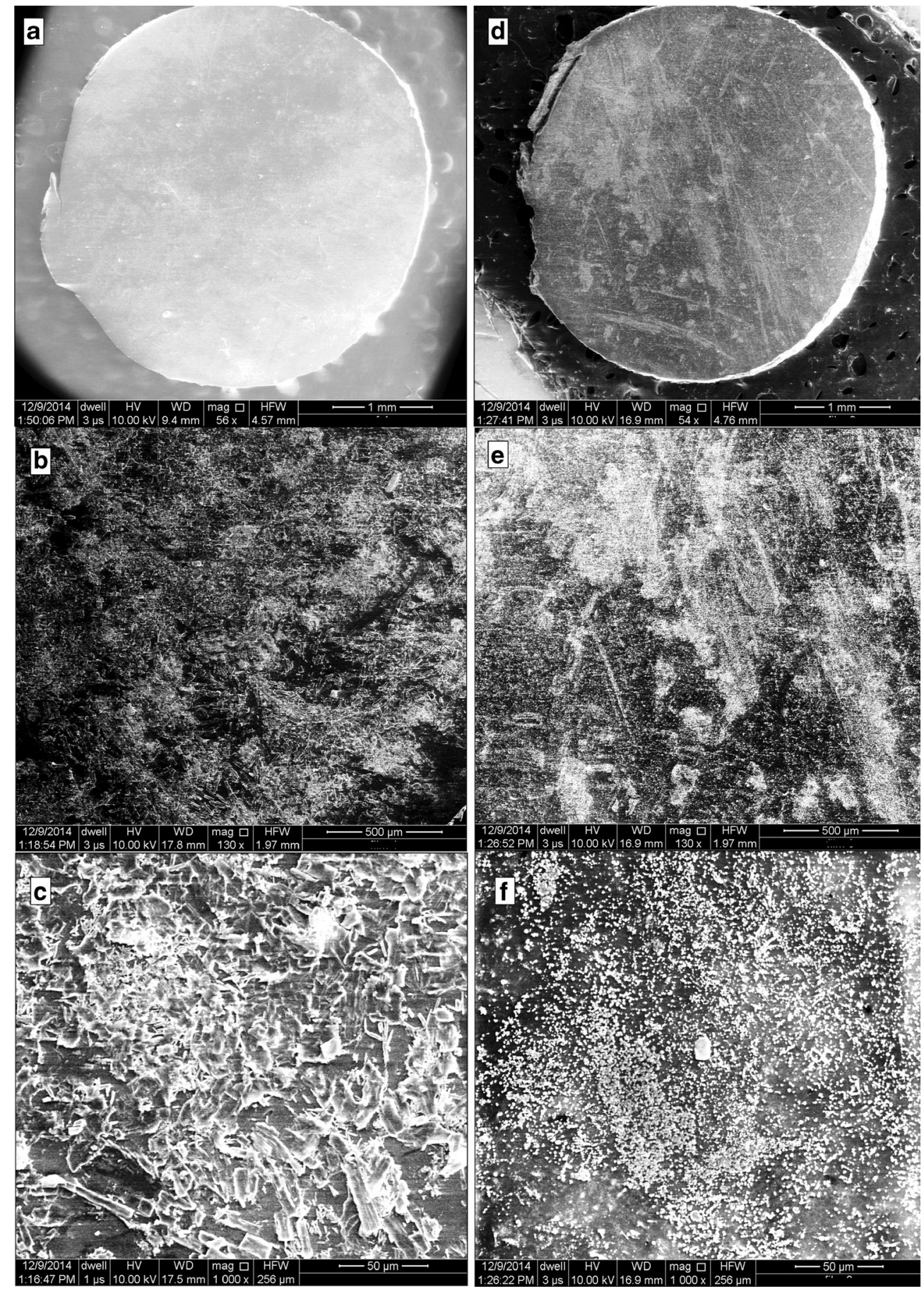

Fig. 5. Scanning electron microscopic images showing surface morphology of a-c placebo films and $\mathbf{d}-\mathbf{f}$ drug-loaded films at different resolutions

\section{Swelling Studies}

Natural polymers like chitosan shows swelling behaviour due to relaxation and interpenetration of polymer chains when in contact with aqueous media. Swelling is an important parameter for drug release in case of natural polymers which shows swelling-controlled release. Further excess swelling of polymeric matrix may loosen the networks causing early release of entrapped drug and decreased mucoadhesion and retention due to the formation of slippery mucilage. In addition, swelled films may ooze out from the pockets causing discomfort to the patient. The swelling behaviour of selected periodontal films as a function of time is illustrated in Fig. 4. Swelling is higher in case of non-crosslinked (F-7, F-8, and F-9) batches as compared to glutaraldehyde crosslinked batches (F-10, F-11). Amongst non-crosslinked batches, a significant effect of plasticizer concentration was observed. Increase in the concentration of plasticizer enhances the 


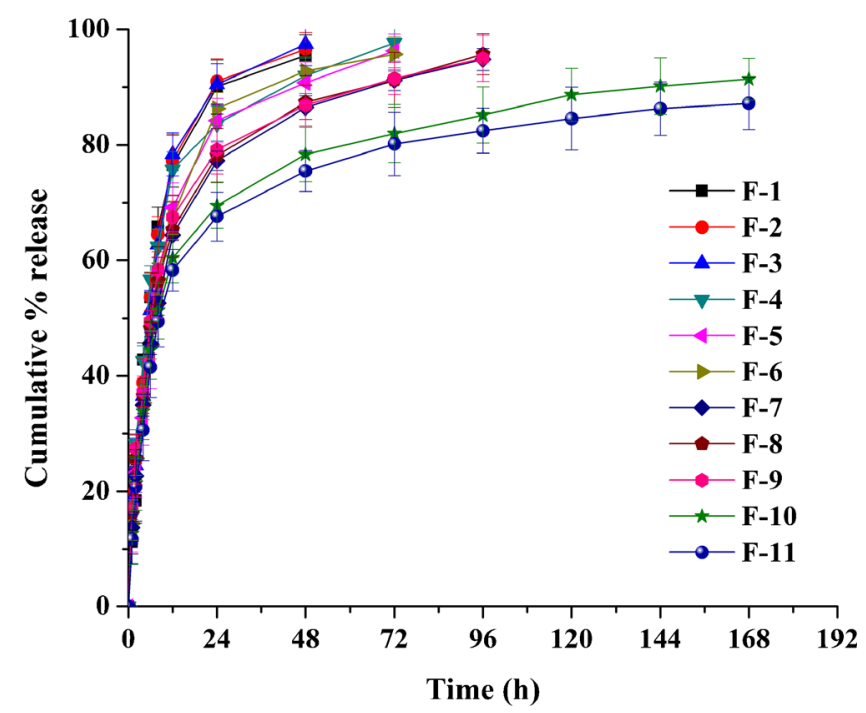

Fig. 6. Cumulative percentage release of MZ from films in McIlvaine buffer $\mathrm{pH} 6.6$ (vertical bars represent mean $\pm \mathrm{SD}, n=3$ )

hydrophilic power of films leading to more swelling viz F-9 > F$8>$ F-7. On contact with water, the plasticizer gets dissolved creating porous films and hence making easy entry of water for drug dissolution. Therefore, swelling plays important role in drug release.

While on the contrary, reduced swelling was observed with an increase in the concentration of crosslinking agent and vice versa. The effect may be attributed to the increase in ionic network formation between positively charged $-\mathrm{NH}_{3}{ }^{+}$ group of chitosan and negatively charged $-\mathrm{C}=\mathrm{O}-$ group of glutaraldehyde. Such ionic networks are strongly bound and act as barrier for polymer swelling. Hence, higher concentration of crosslinking agent minimizes the degree of swelling (30). Batch F-11 showed minimum swelling due to the presence of higher amount (5\%) of glutaraldehyde.

\section{Surface Morphology of Selected Batch}

The surface morphology of the drug-loaded film was assessed using scanning electron microscopy and shown in Fig. 5. The reason for selection of F-11 batch amongst all the batches is its quality in every aspect such as folding endurance, surface $\mathrm{pH}$, and its in vitro release profile. SEM images were taken at different magnifications 54X, 56X, 130X and 1000X to investigate the surface of films. The film surface appeared smooth and compact with no apparent pores smooth at lower magnifications (54X and 56X), while at higher magnifications (1000X) the presence of drug crystals in case of drug-loaded films can be observed on the surface of the films. This adhered drug on the surface of film could be responsible for the burst effect in the initial stage. Considering the initial pathological

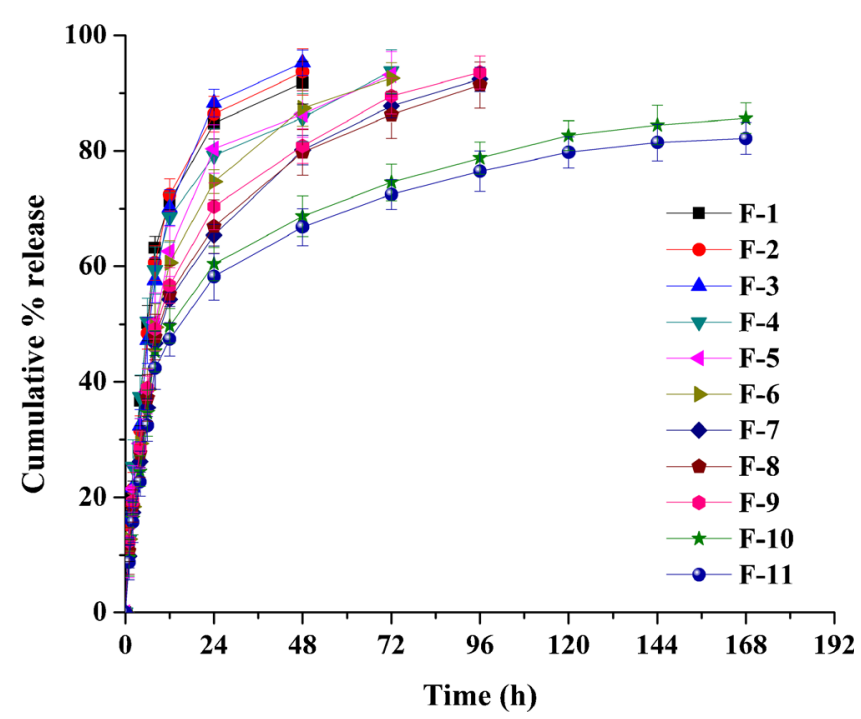

Fig. 7. Cumulative percentage release of LF from films in Mcllvaine buffer $\mathrm{pH} 6.6$ (vertical bars represent mean $\pm \mathrm{SD}, n=3$ ) 
Table III. Kinetic Modelling for Optimized Batch

\begin{tabular}{|c|c|c|c|c|c|c|c|c|c|c|}
\hline \multirow[t]{3}{*}{ Batch code } & \multicolumn{6}{|c|}{$r^{2}$ value } & \multicolumn{4}{|c|}{ Korsemeyer-Peppas model } \\
\hline & \multicolumn{2}{|c|}{ Zero order } & \multicolumn{2}{|c|}{ First order } & \multicolumn{2}{|c|}{ Higuchi model } & \multicolumn{2}{|c|}{$r^{2}$ value } & \multicolumn{2}{|c|}{$n$ value } \\
\hline & $\mathrm{MZ}$ & LF & $\mathrm{MZ}$ & LF & MZ & $\mathrm{LF}$ & $\mathrm{MZ}$ & $\mathrm{LF}$ & $\mathrm{MZ}$ & $\mathrm{LF}$ \\
\hline $\mathrm{F}-11$ & 0.65 & 0.70 & 0.82 & 0.83 & 0.85 & 0.88 & 0.99 & 0.97 & 0.641 & 0.624 \\
\hline
\end{tabular}

load in the periodontal pockets, an initial burst release of the antimicrobial is desirable.

\section{In Vitro Release Study}

Drug release from films was evaluated in McIlvaine buffer pH 6.6 for about $168 \mathrm{~h}$ by static method as described earlier. The periodontal films released the drug in a biphasic manner with an initial burst release followed by sustained release up to 7 days (Figs. 6 and 7). The immediate burst release is contributed by the leaching of free drug particles on the superficial layer of the film which were at immediate access to the dissolution media. On the account of presence of bacterial load in pockets and to provide immediate effect and achieve MIC, burst release of antibiotics is useful for antimicrobial therapy. The cumulative percent drug release (CPR) depends on the composition of films; $80 \%$ of CPR has been observed from non-crosslinked batches in $24 \mathrm{~h}$ whereas crosslinked batches delivered less than $60 \%$ only in $24 \mathrm{~h}$. The remaining portion of drug followed sustained release pattern of drug release up to 4 days in case of non-crosslinked batches and for 7 days in case of crosslinked batches. A similar kind of release pattern was reported in previous studies conducted by Barat et al. and Kassem et al. (31,32). The kinetic data of optimized batch with $r^{2}$ values obtained for different models is depicted in Table III. In vitro kinetic analysis showed that drug release was best explained by KorsemeyerPeppas equation than other models, with the highest value of linearity $\left(r^{2}>0.9\right)$ for all formulations. The diffusion coefficient (n) value for Korsemeyer-Peppas equation for all batches was more than 0.5 which showed drug release mechanism to be combined effect of erosion and diffusion. This is expected, as chitosan is hydrophilic, water swellable and biodegradable.

Further, release studies showed that the ratio of drug and polymer, plasticizer concentration and crosslinking have significant effect on cumulative percent release of both the drugs. The release is slower and more sustained following the slight increase in CS concentration due to increase in matrix density and path length. Contrarily, on increasing plasticizer concentration the drug release was faster. The change in the release rate was possibly due to the alteration in membrane permeability caused by the modification of film hydrophilicity by the plasticizer (33). The slower or faster drug release can be expressed in terms of $\mathrm{T}_{90}$. The higher $\mathrm{T}_{90}$ indicates that more time is required to release $90 \%$ of drug from the matrix, hence slower the release and vice versa. The higher concentration of chitosan and crosslinking agent has positively affected $\mathrm{T}_{90}$ of both the drugs while plasticizer concentration showed negative effect on $\mathrm{T}_{90}$. Lower concentration of polymer demonstrated faster release due to less quantity of drug entrapped inside the polymer matrix in comparison to the superficial layer of the film. When drug-loaded film comes in contact with release medium, the drug from the surface leaches into the surrounding medium and makes the more porous polymeric film structure which in turn enables faster drug diffusion from the matrix and thereby decreasing the $\mathrm{T}_{90}(17)$.

Amongst non-crosslinked films, batch F-7 demonstrated optimum sustained-release profile for $96 \mathrm{~h}$ with higher value of $\mathrm{T}_{90}$ considering largest quantity of $\mathrm{CS}$ and lowest quantity

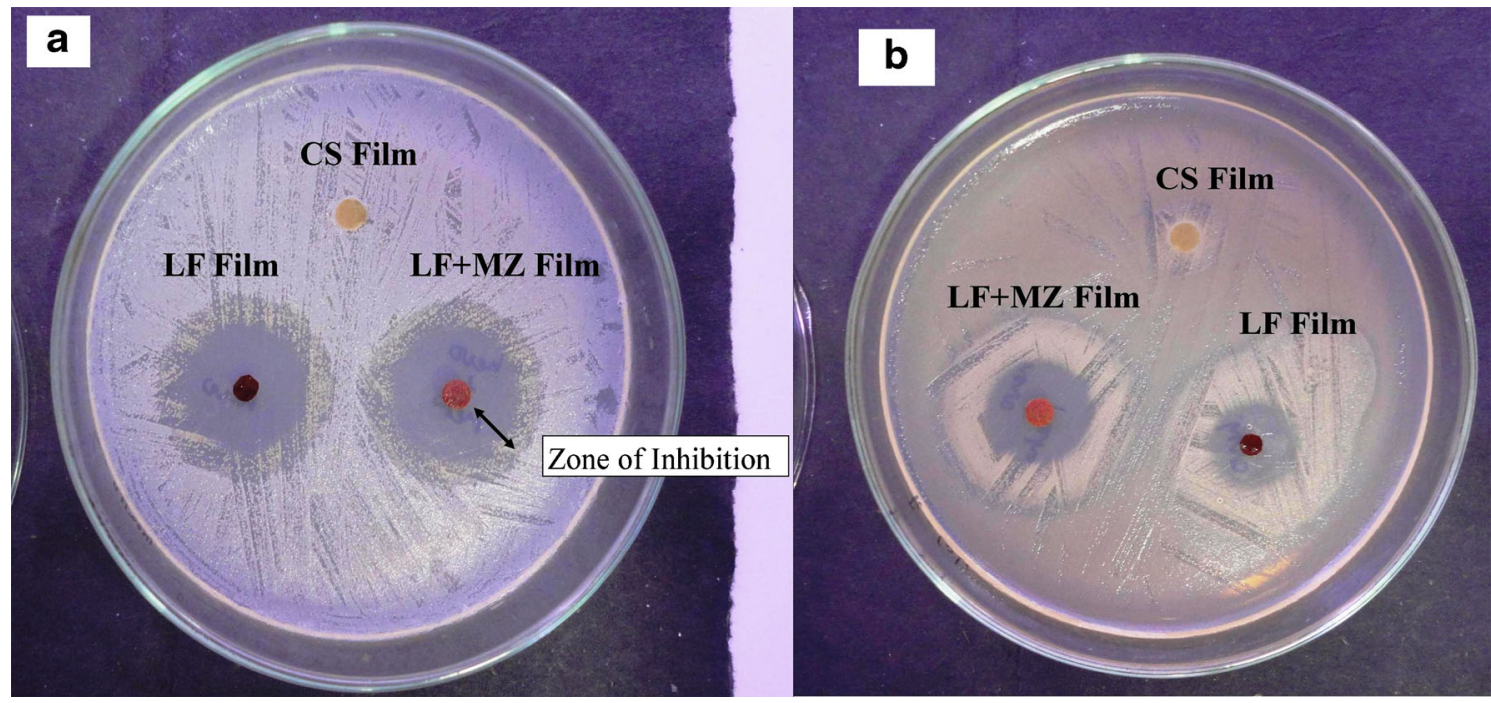

Fig. 8. Inhibition of bacterial growth on agar plate by CS film, LF film and LF + MZ film against a S. aureus and $\mathbf{b} E$. coli 

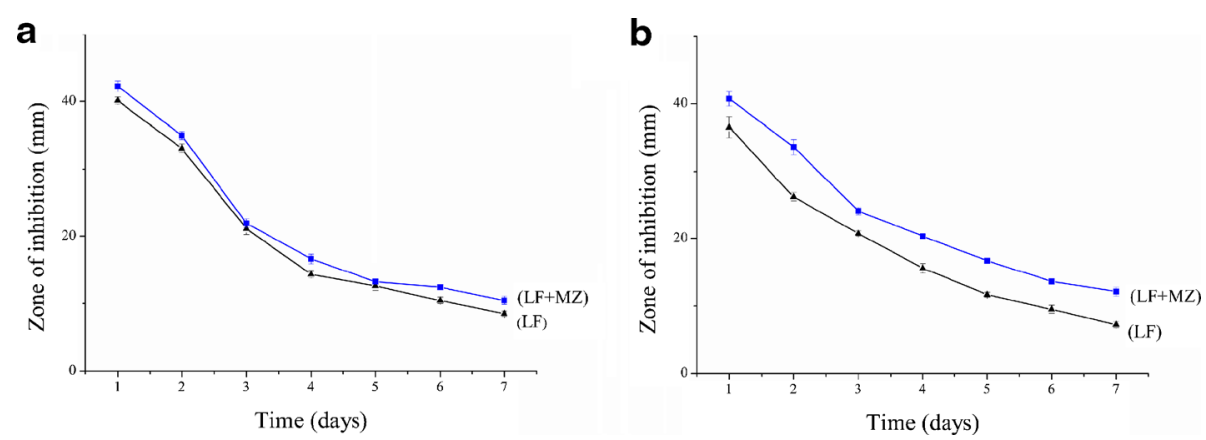

Fig. 9. The inhibition zones of $\mathrm{LF}+\mathrm{MZ}$ and $\mathrm{LF}$ films against a $S$. aureus and $\mathbf{b}$ E. coli for 7 days (vertical bars represent mean $\pm \mathrm{SD}, n=3$ )

of plasticizer. The batch was further selected and crosslinked with different concentrations of glutaraldehyde $2.5 \%$ (F-10) and $5 \%(\mathrm{~F}-11)$. With high concentration of glutaraldehyde, F-11 showed lower burst release and more sustained effect for up to 7 days than F-10.

The crosslinked film showed higher value of $\mathrm{T}_{90}$ as compared to the non-crosslinked film. The significant decrease in the drug release rate and thereby increase in $\mathrm{T}_{90}$ was attributed to the decrease in the porous structure. Thereby, the permeability coefficient of the crosslinked film resulted from the crosslinking between the amino group of CS and aldehyde group of glutaraldehyde (34). Therefore, F-11 batch was selected as the optimum batch for antibacterial and clinical study not only on the basis of release profile but also on the basis of other physicochemical properties like swelling index and burst release. In conducting similarity and dissimilarity studies, nonsignificant difference was found between F-10 and F-11 batch, but F-11 showed minimum burst release and minimum swelling in the buffer. Further, burst release is desirable but excess of burst release above the MIC of the drug is undesirable and leads to wastage of drug. Moreover, minimum swelling of films is required so as they maintain their shape and integrity within the periodontal pocket, which is desirable for the treatment of periodontitis.

\section{Stability Studies}

After storage, no significant changes in colour and texture of the film were observed. The drug content, swelling index and in vitro release profile of stored films were comparable and were within limits. Further, FTIR spectra of stored film and freshly prepared films can be superimposed, indicating the stability of the films (Fig. 2). Hence, the films can be used after storage of 3 months without any loss of physical and chemical attributes.

\section{In Vitro Antibacterial Activity}

The antibacterial activity of the films was estimated by measuring zone of inhibition against $S$. aureus (ATCC25323) and E. coli (ATCC25922). Zone of inhibition was calculated for optimized crosslinked polymeric film (batch F-11) containing $\mathrm{LF}+\mathrm{MZ}$, placebo film and LF film at every day until 7 days (Fig. 8). LF $+\mathrm{MZ}$ film showed higher mean inhibition zone of $42.26 \pm 0.40 \mathrm{~mm}$ and $40.76 \pm 1.10 \mathrm{~mm}$ for $S$. aureus and $E$. coli respectively on the first day and then it slowly decreased to $10.5 \pm 0.50 \mathrm{~mm}$ and $12.13 \pm 0.66 \mathrm{~mm}$ after 7 days of incubation
(Fig. 9). LF films exhibited good zone of inhibition against $S$. aureus with value of $40.1 \pm 0.52 \mathrm{~mm}$ whereas lesser activity for $E$. coli having zone of inhibition of $36.53 \pm 1.55 \mathrm{~mm}$ on the first day of the assay and then it slowly decreased to $8.5 \pm$ $0.40 \mathrm{~mm}$ and $7.24 \pm 0.48 \mathrm{~mm}$ after 7 days of incubation (Fig. 9). Moreover, CS films showed very mild activity against $S$. aureus and $E$. coli on the first day only. No further activity was observed on subsequent days. These effects were previously demonstrated by other workers (35).

Thus, it can be inferred that the combination of the drugs $\mathrm{MZ}$ and LF has provided better activity against Gram-positive as well as Gram-negative bacteria in comparison to single drug (LF) film. Further, the films maintained their antibacterial effect for 7 days, and loading of LF and MZ into polymer matrix has not affected the antibacterial activity of individual drugs. In addition to drugs, little antimicrobial activity can also be attributed to presence of CS.

\section{Clinical Study}

Clinical study was conducted to assess the efficacy and therapeutic potential of MZ and LF containing film in the patients suffering from the chronic periodontitis. Furthermore, its potential was compared with only SRP (group 1), SRP + CS film (group 2), SRP + LF film (group 3 ) and SRP $+M Z$ and LF film (group 4). All treatments were given to the patient at four sites to minimize the influence of the risk factors related to periodontal disease on the outcomes of the treatment provided. Periodontal disease is a multifactorial disease in nature. There are many risk factors such as genetic, socioeconomic status, age, gender, oral hygiene maintenance, host immune defence mechanism, and stress. Ultimately, the progression of periodontal disease and outcome of the treatment depend upon the interaction between the microorganisms and host-related factors.

The films were inserted in the periodontal pockets of the group of patients after scaling and root planning i.e. at the baseline (Fig. 10). The patients were recalled every week for check-up during the total study period of 8 weeks. The treated sites in groups 2, 3 and 4 did not show any redness, swelling or pain; however, some patients complained of mild discomfort at the treated sites for 1 or 2 days. Apart, no complaints regarding inflammation, irritation or pus formation were reported during the whole period of study, which indicated that the films were compatible and tolerable to patients.

The results of the clinical study are presented for all groups as mean reduction of the tested parameters following 

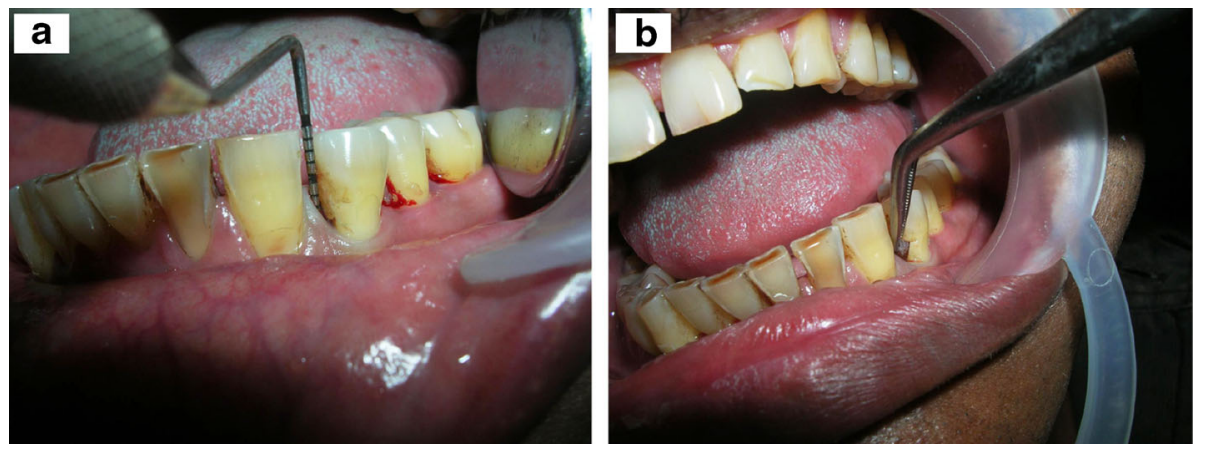

Fig. 10. Clinical application of film: a pocket depth measurement and $\mathbf{b}$ film application

8 weeks of treatment relative to the baseline values (Fig. 11). The significant reduction $(p<0.05$ and $p<0.001)$ in the PPD was observed for all the drug-treated groups (group 3 and group 4) from the baseline value upon recalled visit compared to group 2. Nevertheless, the reduction in the PPD for group 4 was highest amongst the entire treated groups $(p<0.001)$ at the end of eighth week (Fig. 11a) after treatment.

The GI which indicates the compliance of the patient towards the periodontal therapy was recorded at regular predefined interval (Fig. 11b). The reduction of GI was found to be statistically significant in all treated groups from the baseline value at second, fourth and eighth week except at the first week. Further, on the second week onwards, groups 3 and 4 showed significant reduction in GI score $(p<0.05$, $p<0.01$ and $p<0.001$ ) as compared to group 2. At the end of the study (after 8 weeks), group 4 showed minimized value of GI amongst all other treated groups, indicating the regaining of oral hygiene in patients treated with MZ + LF films.

Similarly, the CAL also showed significant reduction during the study span (Fig. 11c). Amongst all, group 4 showed significant $(p<0.05$ and $p<0.001)$ reduction in CAL from fourth week onwards. However, the reduction in CAL for group 4 was higher than group 3 after 8 weeks, which confirms the enhancement of therapeutic efficacy due to strong a

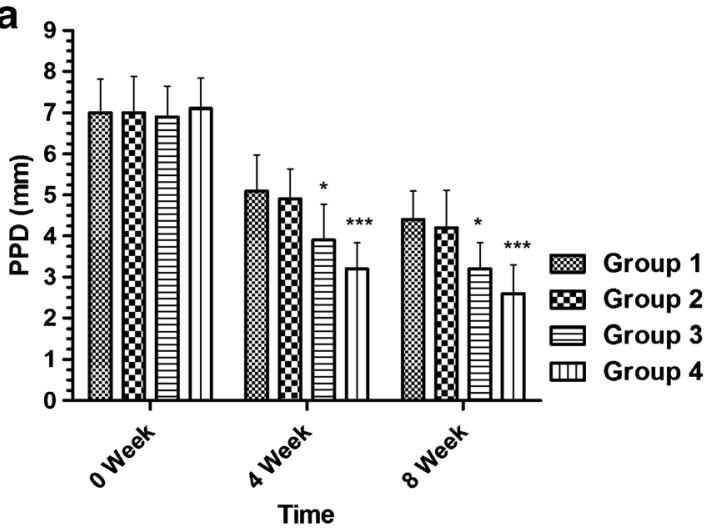

C

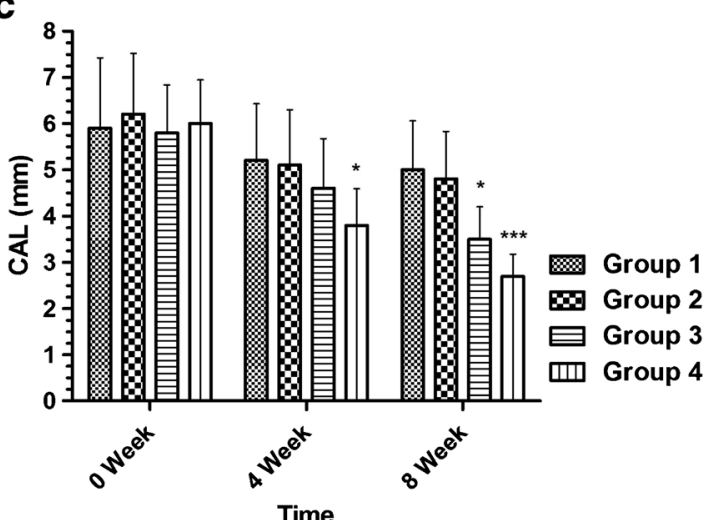

b

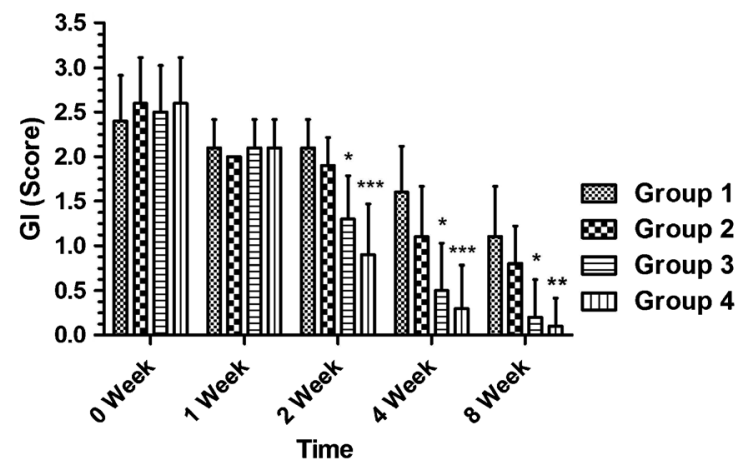

d

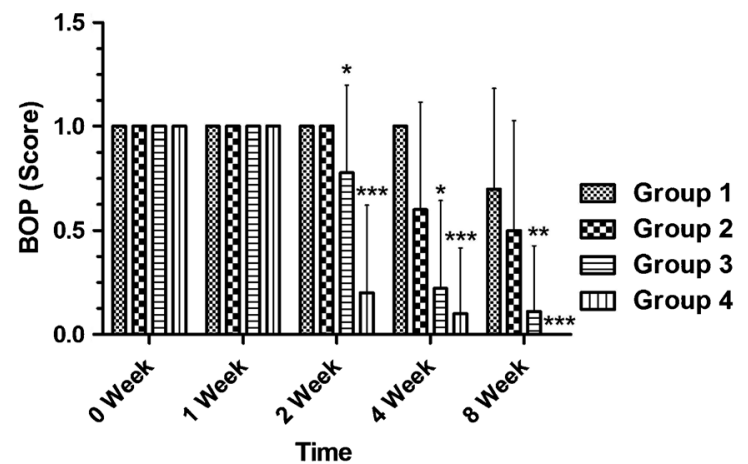

Fig. 11. Values are mean \pm SD for each group during clinical evaluation of following parameters obtained after 8 weeks. a Probing pocket depth (PPD). b Score of gingival index (GI). c Clinical attachment level (CAL). d Score of bleeding on probing (BOP). Scores of GI: 0 no need for periodontal treatment, 1 oral hygiene instruction (OHI), 2 OHI, removal of plaque retentive factors including all supra- and subgingival calculus, $3 \mathrm{OHI}$, root surface debridement (RSD). Scores of BOP: 0 no bleeding and 1 bleeding on probing. ${ }^{*} p<0.05, * * p<0.01$ and $* * * p<0.001$ compared to group 2 by two-way (ANOVA) 
combined antimicrobial effect of both drugs in the single delivery device (film) on the periodontal pathogens as being previously reported in a similar study (36).

The findings of BOP revealed that significant $(p<0.05$, $p<0.01$ and $p<0.001)$ reduction in BOP was observed for groups 3 and 4, from the second week onwards compared to group 2 (Fig. 11d). After the fourth week of study, BOP was not observed for group 4 whereas other groups showed BOP which establishes the therapeutic potential of the developed film.

At the end of study, a significant reduction in pocket depth, GI, bleeding on probing and reattachment of periodontal ligament was observed for groups 3 and 4 . However, the reduction of all studied parameters for group 4 was higher compared to group 3. The obtained results showed that the treatment period had a significant effect on the mean values of the tested clinical parameters for group 4 from the baseline.

From the clinical study, it could be concluded that the films were well tolerated and no sign of irritation was observed at the place of treatment. Although the traditional technique, SRP, applied for the treatment of periodontal pockets is effective as being previously reported (37), group 4 treated with SRP along with the combination drug therapy confirmed higher improvement compared to the group 1 treated with SRP alone. After treatment of 8 weeks, the gum turned into pale pink having no signs of swelling, inflammation along with swift reattachment of periodontal ligament and significant reduction in pocket depth. Therefore, MZ $+\mathrm{LF}$ films were more effective in the management of periodontal health as compared to LF films or SRP alone.

\section{CONCLUSION}

The present study succeeded in the fabrication of biodegradable, mucoadhesive film of $\mathrm{CS}$ by solvent casting. Processing factors such as CS, plasticizer and crosslinking agent concentration have significantly affected the physical characteristics of film and were found within acceptable range. CS concentration has negatively affected drug release and positively affected $\mathrm{T}_{90}$ due to altered matrix density. On the contrary, plasticizer concentration has positively affected the rate of drug release and negatively affected $\mathrm{T}_{90}$ of both drugs attributed to increased membrane permeability. The release of drugs from crosslinked films showed sustained release for 7 days in comparison with non-crosslinked film because of its decreased permeability coefficient. The use of combination of drugs has proven to surpass the potential over single drug film and placebo film during antibacterial study and clinical study. The local delivery of both drugs in a sustained release formula enhances the therapeutic effect of SRP as demonstrated by the measured clinical parameters. Conclusively, such cheap, less resource-requiring film devices have great market potential to administer medicament locally into the periodontal pockets for the management of periodontitis.

\section{ACKNOWLEDGMENTS}

The first author is thankful to UGC, New Delhi, for providing funds for conducting the study. All authors are thankful to Mr. Mayank Gangwar, Institute of Medical Sciences, Banaras Hindu University, India, for the assistance of antibacterial activity.

\section{COMPLIANCE WITH ETHICAL STANDARDS}

Conflict of Interest

The authors state no conflict of inter-

est between them.

\section{REFERENCES}

1. Labib GS, Aldawsari HM, Badr-Eldin SM. Metronidazole and pentoxifylline films for the local treatment of chronic periodontal pockets: preparation, in vitro evaluation and clinical assessment. Expert Opin Drug Deliv. 2014;11(6):855-65.

2. Li X, Kolltveit KM, Tronstad L, Olsen I. Systemic diseases caused by oral infection. Clin Microbiol Rev. 2000;13(4):547-58.

3. Zamani M, Morshed M, Varshosaz J, Jannesari M. Controlled release of metronidazole benzoate from poly epsiloncaprolactone electrospun nanofibers for periodontal diseases. Eur J Pharm Biopharm. 2010;75:179-85.

4. Jain N, Jain GK, Javed S, Iqbal Z, Talegaonkar S, Ahmad FJ, et al. Recent approaches for the treatment of periodontitis. Drug Discov Today. 2008;13(21-22):932-43.

5. Ellen H, Marianne H, Arne S. Mucoadhesive and drug permeation of free mixed films of pectin and chitosan: an in vivo study. Eur J Pharm Biopharm. 2009;71:325-31.

6. Greenstein G, Polson A. The role of local drug delivery in the management of periodontal diseases: a comprehensive review. J Periodontol. 1998;69(5):507-20.

7. Rinaudo M. Chitin and chitosan: properties and applications. Prog Polym Sci. 2006;31:603-32.

8. Chaubey P, Patel RR, Mishra B. Development and optimization of curcumin-loaded mannosylated chitosan nanoparticles using response surface methodology in the treatment of visceral leishmaniasis. Expert Opin Drug Deliv. 2014;11(8):1163-81.

9. Abruzzo A, Bigucci F, Cerchiara T, Cruciani F, Vitali B, Luppi B. Mucoadhesive chitosan/gelatin films for buccal delivery of propranolol hydrochloride. Carbohydr Polym. 2012;87:581-8.

10. Remunan LC, Bodmeier R. Mechanical and water vapor transmission properties of polysaccharide films. Drug Dev Ind Pharm. 1996;22:1201-9.

11. Amal HE, Lubna YA, Ibrahim AA. Micromatricial metronidazole benzoate film as a local mucoadhesive delivery system for treatment of periodontal diseases. AAPS PharmSciTech. 2007;8(3):E1-11.

12. Perioli L, Ambrogi V, Rubini D, Giovagnoli S, Ricci M, Blasi P, et al. Novel mucoadhesive buccal formulation containing metronidazole for the treatment of periodontal disease. J Control Release. 2004;95(3):521-33.

13. Ogrendik M. Levofloxacin treatment in patients with rheumatoid arthritis receiving methotrexate. South Med J. 2007;100(2):135-9.

14. Credito KL, Jacobs MR, Appelbaum PC. Anti-anaerobic activity of levofloxacin alone and combined with clindamycin and metronidazole. Diagn Microbiol Infect Dis. 2000;38(3):181-3.

15. Bharadwaj R, Vidya A, Dewan B, Pal A. An in vitro study to evaluate the synergistic activity of norfloxacin and metronidazole. Indian J Pharmacol. 2003;35:220-6.

16. Nair AB, Kumria R, Harsha S, Attimarad M, Al-Dhubiab BE, Alhaider IA. In vitro techniques to evaluate buccal films. J Control Release. 2013;166(1):10-21.

17. Barat R, Srinatha A, Pandit JK, Ridhurkar D, Balasubramaniam $\mathrm{J}$, Mittal N, et al. Niridazole biodegradable inserts for local longterm treatment of periodontitis: possible new life for an orphan drug. Drug Deliv. 2006;13(5):365-73.

18. Nafee N, Boraie MA, Ismail FA, Mortada LM. Design and characterization of mucoadhesive buccal patches containing cetylpyridinium chloride. Acta Pharm. 2003;53(3):199-212. 
19. Shi P, Li Y, Zhang L. Fabrication and property of chitosan film, carrying ethylcellulose microspheres. Carbohydr Polym. 2008;72:490-9.

20. Sridevi S, Babu RJ, Mittal N, Kumar DS, Pandit JK. Development and evaluation of acrylic strips of metronidazole for the therapy of periodontal disease. Pharmazie. 1995;50(2):153-4.

21. Tiwari G. Biodegradable monolithic periodontal films for controlled delivery of metronidazole to periodontal pocket. Int J Pharma Bio Sci. 2010;2:1-8.

22. Sharma AK, Gangwar M, Tilak R, Nath G, Sinha ASK, Tripathi $\mathrm{YB}$, et al. Comparative in-vitro antimicrobial and phytochemical evaluation of methanolic extract of root, stem and leaf of Jatropha curcas Linn. Phcog J. 2012;4(30):34-40.

23. Rao SB, Sharma CP. Use of chitosan as a biomaterial: studies on its safety and hemostatic potential. J Biomed Mater Res. 1997;34(1):21-8.

24. Mundargi RC, Srirangarajan S, Agnihotri SA, Patil SA, Ravindra $\mathrm{S}$, Setty SB, et al. Development and evaluation of novel biodegradable microspheres based on poly (d, 1-lactide-co-glycolide) and poly (epsilon-caprolactone) for controlled delivery of doxycycline in the treatment of human periodontal pocket: in vitro and in vivo studies. J Control Release. 2007;119(1):59-68.

25. Ramachandran S, Nandhakumar S, Dhanaraju MD. Formulation and characterization of glutaraldehyde cross-linked chitosan biodegradable microspheres loaded with famotidine. Trop J Pharm Res. 2011;10(3):309-16.

26. $\mathrm{Hu} \mathrm{H}$, Xin JH, Hu H, Chan A, He L. Glutaraldehyde-chitosan and poly vinyl alcohol blends, and fluorescence of their nanosilica composite films. Carbohydr Polym. 2013;91:305-13.

27. Ahuja A, Ali J, Rahman S. Biodegradable periodontal intrapocket device containing metronidazole and amoxycillin: formulation and characterisation. Pharmazie. 2006;61(1):25-9.
28. Suyatma NE, Tighzert L, Copinet A. Effects of hydrophilic plasticizers on mechanical, thermal, and surface properties of chitosan films. J Agric Food Chem. 2005;53:3950-7.

29. Lourdin D, Bizot H, Colonna P. "Antiplasticization" in starchglycerol films. J Appl Polym Sci. 1997;63:1047-53.

30. Macleod GS, Collett JH, Fell JT. The potential use of mixed films of pectin, chitosan and HPMC for bimodal drug release. J Control Release. 1999;58(3):303-10.

31. Barat R, Srinatha A, Pandit JK, Anupurba S, Mittal N. Chitosan inserts for periodontitis: influence of drug loading, plasticizer and crosslinking on in vitro metronidazole release. Acta Pharm. 2007;57(4):469-77.

32. Kassem AA, Ismail FA, Naggar VF, Aboulmagd E. Preparation and evaluation of periodontal films based on polyelectrolyte complex formation. Pharm Dev Technol. 2015;20(3):297-305.

33. Shao ZJ, Moralesi L, Diaz S, Muhammadi NA. Drug release from Kollicoat SR 30D-coated nonpareil beads: evaluation of coating level, plasticizer type, and curing condition. AAPS PharmSciTech. 2002;3(2), E15.

34. Thacharodi D, Rao KP. Development and in vitro evaluation of chitosan-based transdermal drug delivery systems for the controlled delivery of propranolol hydrochloride. Biomaterials. 1995;16(2):145-8.

35. Kong M, Chen XG, Xing K, Park HJ. Antimicrobial properties of chitosan and mode of action: a state of the art review. Int J Food Microbiol. 2010;144:51-63.

36. Salvi GE, Mombelli A, Mayfield L, Rutar A, Suvan J, Garrett S, et al. Local antimicrobial therapy after initial periodontal treatment. J Clin Periodontol. 2002;29(6):540-50.

37. Cobb CM. Non-surgical pocket therapy: mechanical. Ann Periodontal. 1996;1(1):443-90. 